\title{
Análisis y propuesta de mejoramiento energético, económico y de habitabilidad en la edificación educativa pública de la UNS
}

\section{Analysis and proposal of energy, economic and habitability improvement in the UNS public educational facility}

\author{
José Luis Fernández, Lucas Rodríguez, Claudio Genovese \\ Colaboradores: Mario Rasquete, Lucrecia Obiol
}

\begin{abstract}
Carrera de Arquitectura, Departamento de Geografía y Turismo, Universidad Nacional del Sur
\end{abstract} Departamento de Ciencias de la Administración, Universidad Nacional del Sur

jlfernandez.arq@gmail.com, arqlucasgrodriguez@gmail.com, cgenoves@uns.edu.ar

\section{RESUMEN}

Partiendo de los edificios del Departamento de Ciencias de la Administración, UNS, como unidades de análisis, se reconoce que las condiciones mínimas de habitabilidad dependen de un significativo insumo de energía auxiliar; registrando importantes costos económicos y ambientales. Consecuentemente, basados en estudios previos y un Proyecto de Grupos de Investigación (PGI) UNS 2018-2020, se infiere que el mejoramiento de la calidad tecnológica en la envolvente de la edificación existente, asegura beneficios higro-térmicos y ambientales en forma inmediata y beneficios económicos en períodos mediatos. A tal fin, en el presente artículo se describe: el marco de actuación; los objetivos de la investigación; la metodología de trabajo; y el desarrollo de los principales análisis y resultados de la investigación. Como conclusión, se reconoce que la aplicación de las alternativas tecnológico-constructivo en la edilicia existente presenta beneficios para todos los actores involucrados: el Estado, los usuarios y la salud del edificio.

\section{ABSTRACT}

Starting with the Departamento de Ciencias de la Administración's building, UNS, as a unit of analysis, it is detected that the minimum comfort conditions of habitability depend on an important amount of auxiliary energy; resulting this in high economic and environmental costs. Thus, based on previous studies within the PGI (Proyecto de Grupos de Investigación) UNS 2018-2020, it is inferred that enhancing the building's envelop technological quality, ensures instant hygro-thermal and environmental benefits as well as economic benefits in the mid and longterm. This article describes the acting context, research objectives, the working methodology; and the development of the main research analysis and results. It is concluded that the application of technologicalconstructive alternatives in the existent building shows benefits for all involved in the equation: the State, the users and the building's health.

PALABRAS CLAVE: eficiencia energética, habitabilidad, mejoramiento tecnológicoconstructivo

KEYWORDS: Energy efficiency, comfort, technolgical and constructive improvement

FECHA DE RECEPCIÓN: 29/7/2021| FECHA DE ACEPTACIÓN: 7/11/2021

DOI: http://dx.doi.org/10.30972/arq.0185666 
Análisis y propuesta de mejoramiento energético, económico y de habitabilidad en la edificación educativa pública de la UNS

Fernández, Rodríguez, Genovese- Colaboradores: Rasquete, Obiol

\section{INTRODUCCIÓN Y MARCO}

El artículo presenta el desarrollo del proyecto titulado: "Diagnóstico y propuestas de mejoramiento de la eficiencia energética en la edificación educativa pública. Caso de aplicación: Departamento de Ciencias de la Administración, UNS", avalado en el marco de "Proyectos de Grupos de Investigación" (PGI) 2019-2021 de la Universidad Nacional del Sur. En dicho proyecto se propone investigar respecto de la relación entre la demanda energética para climatización en un edificio seleccionado del Departamento de Ciencias de la Administración (DCA) de la Universidad Nacional del Sur (UNS) y el costo económico integral para el Estado, en la obtención de los estándares adecuados de habitabilidad; desde un posicionamiento sustentable para los usuarios y el Estado.

Para ello, se determinan los niveles de habitabilidad validados en nuestro contexto (según Norma IRAM); a partir de lo cual se trabaja en el desarrollo de los dos aspectos consecuentes: i) la calidad constructiva de la edificación (desde el análisis de sus características higro-térmicas y calidad de los sistemas y artefactos de climatización) y ii) los costos económicos (desde la evaluación de la relación entre los costos operativos y los costos de inversión para mejoramiento de la calidad constructiva en función del tiempo).

De esta forma, se aborda el tema de la climatización en los edificios públicos (trabajando sobre el DCA, UNS), proponiendo alternativas validadas que colaboren en la ampliación de los preconceptos respecto del par energía-costo, reconociéndose por un lado que la demanda energética en calefacción y refrigeración puede ser reducida si se mejora la calidad tecnológicoconstructiva de la envolvente (de acuerdo con las normativas vigentes, estimuladas desde la Ley provincial №13059); y por otro lado, comprendiendo que el costo inicial de inversión que insumen tales acciones se recuperan en períodos mediatos, obteniéndose mejoras inmediatas en la calidad de habitabilidad interior y reducción de emisiones contaminantes al ambiente.

\section{OBJETIVOS Y METODOLGÍA}

Teniendo en cuenta los consumos presentes y entendiendo a las ciudades como estructuras energo-intensivas (DISCOLI, 2009) que dependen necesariamente de estos recursos para mantener las condiciones requeridas de confort y habitabilidad, se propone minimizar la demanda de climatización, buscando una relación más eficiente entre el consumo energético creciente y la eficiencia en el uso. Para ello se plantea mejorar las variables constructivas de la envolvente de las edificaciones educativas públicas (muros, aberturas y cubierta), como así también la optimización de los equipos de climatización, permitiendo establecer una relación más equilibrada entre la energía utilizada, la posible sustitución, la habitabilidad y sus consecuencias ambientales.

Consecuentemente, se establece como objetivo general: Analizar, evaluar y proponer acciones para el mejoramiento tecnológico-constructivo de la envolvente edilicia existente a partir de técnicas apropiadas de conservación energética, con el objeto de reducir el consumo energético y su costo económico, mejorar las condiciones de habitabilidad y favorecer la implementación de Energías Renovables (ER).

\section{Metodología de trabajo}

El desarrollo de la investigación presenta tres fases operativas definidas: una primera instancia de diagnósticos (estudio y descripción del escenario de actuación), una segunda instancia de análisis y propuestas de mejoramiento, y una tercera instancia de evaluación y síntesis. A partir de lo cual se definen las estrategias y herramientas necesarias para i) el relevamiento, ii) la evaluación termo-energética y iii) la evaluación económica. 
Desde esta lógica, se reconoce la necesidad de trabajar en forma conjunta y complementaria desde aportes de los grupos de investigación de la carrera de Arquitectura del Departamento de Geografía y Turismo y del Departamento de Ciencias de la Administración, a fin de presentar propuestas y acciones de calidad en beneficio de la institución y los propios actores que conforman la Universidad Nacional del Sur.

\section{i) Relevamiento:}

El relevamiento de las dimensiones y las características tecnológico-constructivas del edificio del Departamento de Ciencias de la Administración a tratar se lleva a cabo mediante la recuperación de los documentos gráficos y su corroboración desde trabajo de campo, seguido de una instancia de gabinete donde se transcribe y/o ajusta la información relevada en los formatos gráficos digitales (archivo tipo AutoCAD) y se confecciona un informe técnico sobre las características constructivas. Respecto de los aspectos de consumos energéticos y niveles de habitabilidad, se recolectan, estudian, analizan y clasifican los datos pertinentes según fuentes validadas.

\section{ii) Evaluación termo-energética:}

Acorde a la problemática planteada y los objetivos específicos, se comprende que la metodología de evaluación termo-energética debe ser ágil, replicable y validada en el contexto nacional. Por lo tanto, se decidió optar por los métodos de cálculo estacionario según IRAM en su serie de "Acondicionamiento térmico de edificios", cubriendo paralelamente las exigencias de la vigente Ley №13059 de la Prov. de Bs. As.

De allí es que se construye un protocolo de medición donde se analiza la transmitancia térmica ("K") de cada uno de los elementos que constituyen la envolvente edilicia (muros, techos y aberturas), para el estado base y para el mejorado (según IRAM № 11601, 2001; № 11507-4, 2010). Se analiza el comportamiento energético global del edificio ("G", según IRAM No 11604, 2001), para así obtener su demanda energética anual (“Q”), según IRAM No 11604, 2001) necesaria para alcanzar los niveles de habitabilidad establecidos por normativa (IRAM № 11605, 1996).

\section{iii) Evaluación económica:}

La complejidad de los costos de materialización de las medidas de mejoramiento tecnológicoconstructivo, así como la complejidad de los insumos energéticos en juego, requieren desarrollar estrategias de evaluación económica y tecnológica flexibles y una instrumentación que pondere en términos relativos y específicos evaluaciones termo-económicas de las diferentes medidas a adoptar en un contexto de costos y subsidios complejo. Para ello se considera la incidencia de los costos en las distintas etapas de intervención: el costo inicial de inversión para realizar las mejoras tecnológico-constructivas, el costo operativo para afrontar el combustible consumido según equipo de climatización, el análisis de recuperación de la inversión inicial (estableciendo algunos supuestos para su estimación) y algunos escenarios de rentabilidad, como evaluación de conveniencia netamente económica.

En términos concretos, el comportamiento energético-económico global se determina como la carga térmica anual multiplicada por el tiempo (medido en años). Por su parte, el costo monetario para la situación base se calcula como el costo operativo en función del tiempo $\left(t \mathrm{CO}_{o t}\right)$ y para las opciones de mejoramiento se calcula como el costo inicial de la inversión sumado al costo operativo mejorado en función del tiempo $\left(\mathrm{Cll}+t \mathrm{CO}_{i t}\right)$. A su vez, para obtener una mayor precisión en relación a las condiciones de nuestro contexto actual, se incorporan variaciones de los costos en función del tiempo. Por ello es que se incluye una tasa de inflación (i), anual y constante, para los costos operativos $\left(\mathrm{CO}_{t}(1+\mathrm{i}) \mathrm{j}\right)$; y una tasa de renta $(\mathrm{r})$, anual y constante, para el costo inicial de inversión $\left(\mathrm{CII}(1+\mathrm{r})^{\mathrm{t}}\right)$. 
Análisis y propuesta de mejoramiento energético, económico y de habitabilidad en la edificación educativa pública de la UNS

\section{DESARROLLO}

En este apartado se describe el trabajo desarrollado y los resultados de las tres instancias operativas de la investigación: i) relevamiento y descripción del escenario de actuación, ii) análisis termo-energético y propuestas de mejoramiento, y iii) evaluación económica y síntesis.

\section{Relevamiento y descripción del escenario de actuación}

El procedimiento de relevamiento constó de las instancias de recolección de datos gráficos y técnicos, el croquisado y fotografiado, la medición in situ y la representación gráfica del edificio en general (fig. 1) y los detalles necesarios para los cálculos particulares de pérdidas térmicas.
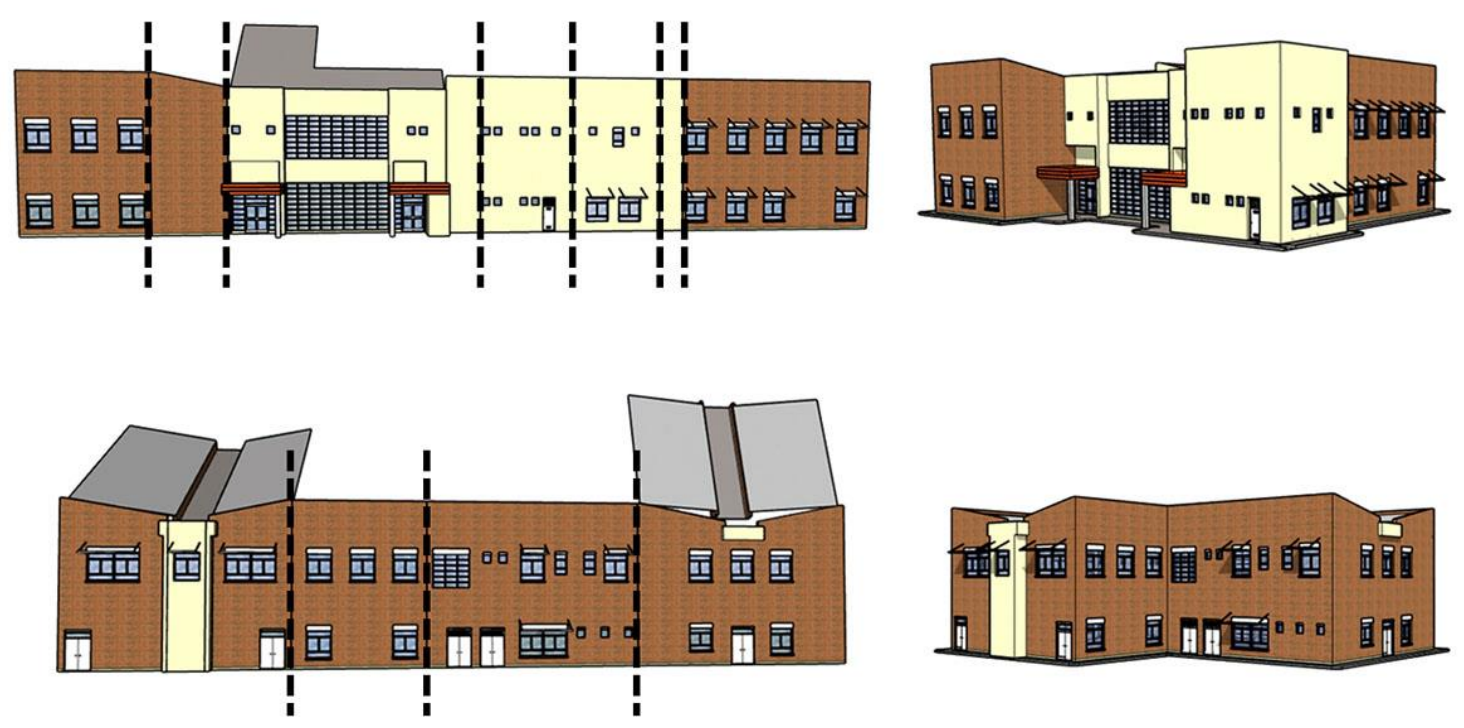

Figura 1. Despiece de la envolvente del edificio. Fuente: elaboración propia

\section{Análisis termo-energético y propuestas de mejoramiento}

En este punto se describe el análisis de la transmitancia térmica de los componentes, el comportamiento energético global y alternativas de mejoramiento tecnológico-constructivo.

\section{a) Análisis de transmitancia térmica de los componentes}

La normativa vigente establece diferentes niveles de habitabilidad (IRAM 11605) para calcular las condiciones ambientales del interior de un edificio, a partir de los valores de transmitancia térmica de los componentes (muros, aberturas y cubierta). En la tabla 1 se describen los valores máximos admisibles para Bahía Blanca (TD -5,6º según IRAM №11.603: 2012).

Tabla 1: Cuadro de niveles de habitabilidad para Bahía Blanca

\begin{tabular}{|l|c|c|c|c|c|}
\hline \multirow{2}{*}{ K adm. max. } & Muros & Cubiertas & Muros & Cubiertas & Grados de \\
& confort \\
\hline Nivel A & $0,30 \mathrm{~W} / \mathrm{m}^{2 \circ} \mathrm{k}$ & $0,26 \mathrm{~W} / \mathrm{m}^{2 \circ} \mathrm{k}$ & $0,50 \mathrm{~W} / \mathrm{m}^{2 \circ} \mathrm{k}$ & $0,19 \mathrm{~W} / \mathrm{m}^{2 \circ} \mathrm{k}$ & Recomendado \\
\hline Nivel B & $0,81 \mathrm{~W} / \mathrm{m}^{2 \circ} \mathrm{k}$ & $0,68 \mathrm{~W} / \mathrm{m}^{2 \circ} \mathrm{k}$ & $1,25 \mathrm{~W} / \mathrm{m}^{2 \circ} \mathrm{k}$ & $0,48 \mathrm{~W} / \mathrm{m}^{2 \circ} \mathrm{k}$ & Medio \\
\hline Nivel C & $1,42 \mathrm{~W} / \mathrm{m}^{2 \circ} \mathrm{k}$ & $1,00 \mathrm{~W} / \mathrm{m}^{2 \circ} \mathrm{k}$ & $2,00 \mathrm{~W} / \mathrm{m}^{2 \circ} \mathrm{k}$ & $0,76 \mathrm{~W} / \mathrm{m}^{2 \circ} \mathrm{k}$ & Mínimo \\
\hline No clasifica & $>1,42 \mathrm{~W} / \mathrm{m}^{2 \circ} \mathrm{k}$ & $>1,00 \mathrm{~W} / \mathrm{m}^{2 \circ} \mathrm{k}$ & $>2,00 \mathrm{~W} / \mathrm{m}^{2 \circ} \mathrm{k}$ & $>0,76 \mathrm{~W} / \mathrm{m}^{2 \circ} \mathrm{k}$ & No clasifica \\
\hline
\end{tabular}

Para el presente estudio se define el nivel $\mathrm{B}$ (medio) como condición de confort higrotérmico mínima, tal como se establece para el cumplimiento de la mencionada Ley 13.059. A tal fin, sobre la base de la aplicación de la norma IRAM, es su serie 11601, 11603, 11605, 11625, 11630, se 
Análisis y propuesta de mejoramiento energético, económico y de habitabilidad en la edificación educativa pública de la UNS

Fernández, Rodríguez, Genovese- Colaboradores: Rasquete, Obiol

describe la calidad tecnológico-constructiva de cada componente y se analiza su transmitancia térmica por unidad de superficie.

i. Muros: se detallan las transmitancias térmicas totales de cada muro tipo, de cada capa componente de las fachadas (fig. 2). Existen en las fachadas dos tipos de muros: A y B. El muro tipo "A", se compone de una cara exterior de ladrillo común visto con junta tomada al ras, ladrillo cerámico hueco de $0,18 \times 0,18 \times 0,33 \mathrm{~m}$, ladrillo cerámico hueco de $0,08 \times 0,18 \times 0,33 \mathrm{~m}$, una capa de hidrófugo y un revoque grueso interior con terminación de yeso. El muro tipo "B" cuenta con las mismas capas que el tipo "A" sin el ladrillo común visto exterior que es reemplazado por un revoque común y revestimiento plástico texturado.

- Fachada Este: es aquella sobre la que se encuentra el acceso público al edificio. Cuenta con dos tipos de muros:

i. Muro tipo A $\left(158,35 \mathrm{~m}^{2}\right)$, transmitancia térmica del componente "K" $1,04 \mathrm{~W} / \mathrm{m}^{2 \circ} \mathrm{K}>$ al recomendado $\left(0,81 \mathrm{~W} / \mathrm{m}^{20} \mathrm{~K}\right)$ : No cumple la normativa. Perdida térmica $\rightarrow 164,68 \mathrm{~W} /{ }^{\circ} \mathrm{K}$

ii. Muro tipo $\mathrm{B}\left(239,09 \mathrm{~m}^{2}\right)$, transmitancia térmica del componente "K" $1,16 \mathrm{~W} / \mathrm{m}^{20} \mathrm{~K}>$ al recomendado $\left(0,81 \mathrm{~W} / \mathrm{m}^{2 \circ} \mathrm{K}\right)$ : No cumple la normativa. Perdida térmica $\rightarrow \mathbf{2 7 7 , 3 4} \mathrm{W} /{ }^{\circ} \mathrm{K}$

- Fachada Norte: Es aquella que enfrenta a la calle principal del campus, la más expuesta al sol. Cuenta con dos tipos de muros:

1. Muro tipo A $\left(151,68 \mathrm{~m}^{2}\right)$, transmitancia térmica del componente " $\mathrm{K}$ " $1,04 \mathrm{~W} / \mathrm{m}^{20} \mathrm{~K}>$ al recomendado $\left(0,81 \mathrm{~W} / \mathrm{m}^{2 \circ} \mathrm{K}\right)$ : No cumple la normativa. Perdida térmica $\rightarrow 157,75 \mathrm{~W} /{ }^{\circ} \mathrm{K}$

2. Muro tipo $B\left(95,4 \mathrm{~m}^{2}\right)$, transmitancia térmica del componente " $\mathrm{K}$ " $1,16 \mathrm{~W} / \mathrm{m}^{2 \circ} \mathrm{K}>$ al recomendado $\left(0,81 \mathrm{~W} / \mathrm{m}^{2} \mathrm{~K}\right)$ : No cumple la normativa. Pérdida térmica $\rightarrow 110,66 \mathrm{~W} /{ }^{\circ} \mathrm{K}$

- Fachada Oeste: Se encuentra frente al área de estacionamiento, opuesta al acceso principal. Cuenta con 2 tipo de muros:

1. Muro tipo $A\left(273,16 \mathrm{~m}^{2}\right)$, transmitancia térmica del componente " $\mathrm{K}$ " $1,04 \mathrm{~W} / \mathrm{m}^{2 \circ} \mathrm{K}>$ al recomendado $\left(0,81 \mathrm{~W} / \mathrm{m}^{2 \circ} \mathrm{K}\right)$ : No cumple la normativa. Perdida térmica $\rightarrow \mathbf{2 8 4 , 0 9} \mathrm{W} /{ }^{\circ} \mathrm{K}$

2. Muro tipo $\mathrm{B}\left(28,71 \mathrm{~m}^{2}\right)$, transmitancia térmica del componente " $\mathrm{K}$ " $1,16 \mathrm{~W} / \mathrm{m}^{2 \circ} \mathrm{K}>$ al recomendado $\left(0,81 \mathrm{~W} / \mathrm{m}^{2 \circ} \mathrm{K}\right)$ : No cumple la normativa. Pérdida térmica $\rightarrow 33,30 \mathrm{~W} /{ }^{\circ} \mathrm{K}$

- Fachada Sur: Enfrenta las partes altas del predio, opuesta a la calle principal del campus, sin ganancia solar directa. Cuenta con un muro tipo de ladrillo visto:

1. Muro tipo A, $\left(249,65 \mathrm{~m}^{2}\right)$, transmitancia térmica del componente " $\mathrm{K}$ " $1,04 \mathrm{~W} / \mathrm{m}^{2 \circ} \mathrm{K}>$ al recomendado $\left(0,81 \mathrm{~W} / \mathrm{m}^{2 \circ} \mathrm{K}\right)$ : No cumple normativa. Perdida térmica $\rightarrow 259,64 \mathrm{~W} /{ }^{\circ} \mathrm{K}$

\section{Perdidas térmicas Totales por muros $\rightarrow 1287,46 \mathrm{~W} /{ }^{\circ} \mathrm{K}$}

A) Muro cerámico doble + ladrillo visto

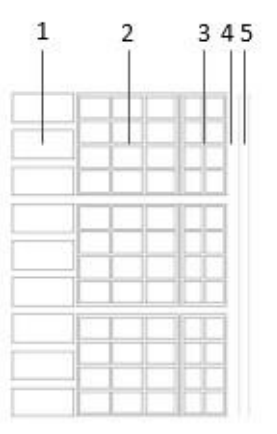

\begin{tabular}{|c|c|c|c|c|}
\hline & MURO & ESPESOR & $\lambda$ & $\mathrm{R}$ \\
\hline & Area total: $158.35 \mathrm{~m}^{2}$ & $\mathrm{~m}$ & W/m.k & $\mathrm{m}^{2} \mathrm{k} / \mathrm{W}$ \\
\hline Res & tencia superficial interior RSI & & & 0.13 \\
\hline 1 & Ladrillo común & 0.11 & 0.91 & 0.121 \\
\hline 2 & Ladrillo hueco 18 & & & 0.41 \\
\hline 3 & Ladrillo hueco 8 & & & 0.23 \\
\hline 4 & Revoque grueso interior & 0.025 & 0.93 & 0.027 \\
\hline 5 & Revoque fino interior & 0.005 & 1.13 & 0.004 \\
\hline Res & tencia superficial exterior RSE & & & 0.04 \\
\hline Esp & or total aproximado & 400 & & \\
\hline RES & STENCIAS TERMICAS TOTALES & & & 0.962 \\
\hline Tran & mitancia térmica del componente & $=1 / R$ & & 1.04 \\
\hline Tran & mitancia Máxima Admisible KMAXA & IRAM 1160 & $3\left[\mathrm{~W} / \mathrm{m}^{2} . \mathrm{k}\right]$ & 0.83 \\
\hline Cun & le con Norma IRAM: SI/NO & & & NO \\
\hline
\end{tabular}

Figura 2. Planilla tipo para la determinación del "K" de los componentes murarios. Ej.: Muro tipo "A". Fuente: elaboración propia 
ii. Carpinterías metálicas: Estas se componen de marcos y hojas de aluminio anodizado blanco de espesor normalizado, con burletería de goma y colizas de felpa, y vidrios comunes float $4 \mathrm{~mm}$. Las ventanas no cuentan con RPT (Ruptura de Puente Térmico). El "K" que corresponde a las carpinterías en estudio es de $5,82 \mathrm{~W} / \mathrm{m}^{2 \circ} \mathrm{K}$, el cual se obtiene según tabla (IRAM 11601, 2002: 30) en correspondencia a vidrio incoloro común. Las condiciones mínimas exigidas por la Ley 13059 para las aberturas se corresponde con los valores de la Norma IRAM 11507-4, estableciéndose que las carpinterías en edificios de hasta $10 \mathrm{~m}$ de altura cuenten con una transmitancia térmica ("K") categoría 3 o $4\left(1.5\right.$ a $2 \mathrm{~W} / \mathrm{m}^{2 \circ} \mathrm{K}$ y de 2 a 3 $\mathrm{W} / \mathrm{m}^{20} \mathrm{~K}$, respectivamente). En consecuencia, ninguna de las aberturas en la envolvente del edificio cumple con la condición de aislación térmica mínima. Las perdidas parciales son:

i. Fachada Este: se computaron $100.19 \mathrm{~m}^{2}$. Pérdida térmica $\rightarrow \mathbf{5 8 2 , 1 1} \mathbf{W} /{ }^{\circ} \mathrm{K}$

ii. Fachada Norte: se computaron $61,86 \mathrm{~m}^{2}$. Pérdida térmica $\rightarrow 360,03 \mathrm{~W} /{ }^{\circ} \mathrm{K}$

iii. Fachada Oeste: se computaron $41,67 \mathrm{~m}^{2}$. Pérdida térmica $\rightarrow \mathbf{2 4 2 , 5 2} \mathbf{W} /{ }^{\circ} \mathrm{K}$

iv. Fachada Sur: se computaron $39,78 \mathrm{~m}^{2}$. Pérdida térmica $\rightarrow 231,52 \mathrm{~W} /{ }^{\circ} \mathrm{K}$

\section{Perdidas térmicas totales por ventanas $\rightarrow 1416,18 \mathrm{~W} /{ }^{\circ} \mathrm{K}$}

iii. Cubierta de techos: La cubierta de techos del edificio se compone de dos grandes paños de chapa ondulada galvanizada $N^{\circ} 25$ que desaguan sobre una losa que hace las veces de canalón y que conduce las aguas de lluvia hacia los desagües pluviales. La chapa de acero galvanizado se apoya sobre una estructura metálica de correas "C" galvanizadas, que sostienen además una manta aislante de $10 \mathrm{~mm}$ doble aluminizada de poliestireno. Debajo de este sistema aparece una cámara de aire que toma diferentes alturas según las pendientes y luego, en contacto con los ambientes habitables, aparece el cielorraso. En algunas ocasiones este es de placas de yeso en módulos de 60x60 y en otros de placa de yeso con junta tomada (manteniendo el mismo espesor, y consecuentemente, la misma transmitancia térmica). El "K" resulta entonces en $1,69 \mathrm{~W} / \mathrm{m}^{2 \circ} \mathrm{K}$ (invierno) y $1,36 \mathrm{~W} / \mathrm{m}^{2 \circ} \mathrm{K}$ (verano). La transmitancia térmica máxima admisible para cubierta, según IRAM 11605 (Nivel B) es de 0,68 W/m ${ }^{2 \circ} \mathrm{K}$ (invierno) y $0,48 \mathrm{~W} / \mathrm{m}^{2 \circ} \mathrm{K}$ (verano). Por lo tanto, la cubierta de techos no cumple con el valor de la normativa en condición de invierno ni verano.

La superficie total de la cubierta de techos es: $\pm 587 \mathrm{~m}^{2}$.

Pérdidas térmicas por cubierta (condición de invierno) $\rightarrow 992,03 \mathrm{~W} /{ }^{\circ} \mathrm{K}$

\section{b) Comportamiento energético global del edificio}

La sumatoria de las pérdidas térmicas de la envolvente del edificio es $3695,67 \mathrm{~W} /{ }^{\circ} \mathrm{K}$. Esto debe entenderse como la energía a inyectar al edificio por cada grado de temperatura por debajo de la temperatura base de confort que se establezca (siendo valores nominales las temperaturas entre 18 y 22 grados). A modo de referencia, se hace mención que las perdidas térmicas del edificio, si este se ajustara a la normativa, es decir utilizando los $K$ mínimos admisibles para cada componente, serían $2098,45 \mathrm{~W} /{ }^{\circ} \mathrm{K}$.

En síntesis, en la actualidad el edificio cuenta con una pérdida térmica por unidad de superficie de $\mathbf{4 3 , 2 2 \%}$ mayor a lo que perdería en caso de ajustarse a los estándares mínimos teóricos. Asumiendo que toda perdida térmica es reemplazada por energía inyectada para mantener el confort interior, se infiere que podrían producirse reducciones considerables en el consumo energético.

Continuando con el análisis termo-energético, se calcula la carga térmica de calefacción anual (Q) de acuerdo al modelo estacionario propuesto (IRAM 11604). Para ello, primero se calcula el coeficiente global de pérdidas térmicas $(G)$, adoptando las 2 renovaciones de aire por hora sugeridas en la Norma. En su desarrollo, este valor se obtiene de sumar las pérdidas térmicas totales (perdidas de muros: $1287,46 \mathrm{~W} /{ }^{\circ} \mathrm{K}$, aberturas: $1416,18 \mathrm{~W} /{ }^{\circ} \mathrm{K}$, cubierta: $992,03 \mathrm{~W} /{ }^{\circ} \mathrm{K}$ y de 
perímetro del suelo: $\left.175,99 \mathrm{~W} /{ }^{\circ} \mathrm{K}\right)$ divididas en el volumen del edificio $\left(2732 \mathrm{~m}^{3}\right)$ más la constante 0,35 (asumida como la capacidad específica del aire) por las renovaciones de aire (2 renovaciones por hora). El " $\mathrm{G}$ " calculado es de $2,12 \mathrm{~W} / \mathrm{m}^{30} \mathrm{~K}$ (a título ampliatorio, se menciona que el " $G$ " admisible para un edificio de estas características es de $1,18 \mathrm{~W} / \mathrm{m}^{3 \circ} \mathrm{K}$ ).

Para el cálculo de la carga térmica anual, se establece una temperatura base de $20^{\circ} \mathrm{C}$, con sus consecuentes 1966 Grados Día (IRAM 11603, 2012). Para su obtención, se multiplica el tiempo de calefacción por día (la norma asume el valor de "24", lo cual concuerda con el caso de estudio), con los grados día de calefacción anual (1966 GD), el "G" calculado $\left(2,12 \mathrm{~W} / \mathrm{m}^{3 \circ} \mathrm{K}\right)$ y el volumen interior del edificio $\left(2732 \mathrm{~m}^{3}\right)$, todo sobre 1000 . El resultado es de $\mathbf{2 7 2 9 1 5} \mathbf{K w h}$ de consumo.

A su vez, este valor debe ser adaptado al rendimiento del equipo de climatización, en caso de maximizar considerablemente el consumo. En el edificio de estudio se cuenta con equipo de radiadores alimentados por caldera a gas natural (GN) de alto rendimiento, cuyos valores se establecen en el orden al $100 \%$. Por tal motivo, el consumo de energía auxiliar suministrada equivale a la energía calórica entregada. Considerando el valor equivalente de poder específico de un $\mathrm{m}^{3}$ de GN a 11,70Kwh, puede inferirse que el edificio presenta un consumo anual de 23326 $\mathbf{m}^{3}$ de GN para alcanzar el nivel mínimo de confort, cubriendo las pérdidas térmicas ocasionadas por la calidad de la envolvente edilicia.

\section{c) Alternativas de mejoramiento tecnológico-constructivo}

A partir del análisis termo-energético, los criterios básicos expresados, la bibliografía base utilizada para los cálculos, la normativa de aplicación, la experiencia profesional en arquitectura de los autores sobre la complejidad de determinados tipos de intervenciones edilicias, y los trabajos de investigación del autor previamente desarrollados en la temática (RODRIGUEZ et al., 2017) se establecen las siguientes alternativas de intervención:

Alternativa 1: Se centra en el tratamiento sobre las carpinterías. Se propone modificar al sistema existente, ventanas corredizas, por una hoja corrediza y paño fijo, ambos paños vidriados con DVH (4+9+4), Línea Módena. El recambio completo de las carpinterías resulta en un alto costo de obra (porque demandaría tareas de mampostería en altura). Por ello, la propuesta considera no intervenir aquello que está fijo al muro (premarcos y marcos) y solo recambiar los paños vidriados (hojas corredizas o paños fijos). La reducción de la transmitancia térmica presentaría un valor de $3,20 \mathrm{~W} / \mathrm{m}^{2 \circ} \mathrm{K}$, resultando en una perdida térmica total de $779,20 \mathrm{~W} /{ }^{\circ} \mathrm{K}$, es decir un $45 \%$ menos que las perdidas actuales por superficies vidriadas.

De esta forma, la transmitancia térmica se reduciría a un valor admisible según IRAM $(3,20$ $\mathrm{W} / \mathrm{m}^{2}{ }^{2} \mathrm{~K}$ equivalente a carpintería tipo $\mathrm{K} 5$, considerando la tolerancia) y el "G" resultaría en 1,91 $\mathrm{W} / \mathrm{m}^{3 \circ} \mathrm{K}$. La carga térmica anual "Q" tendría un valor de $242860 \mathrm{Kwh}$ (o su equivalente de $20757 m^{3}$ de GN), obteniéndose una disminución del consumo total del orden de $11 \%$.

Alternativa 2: Se propone la intervención de todas las fachadas, reduciendo las pérdidas térmicas calculadas y mejorando, consecuentemente, la condición de verano por atenuar las ganancias solares directas. A tal fin, se propone la siguiente opción tecnológico-constructiva:

Aplicación de placa de poliestireno expandido densidad estándar (EPS) de $2 \mathrm{~cm}$ de espesor en todas las superficies interiores de los muros, placa de yeso de $12,5 \mathrm{~mm}$ (tipo Durlock) como revestimiento y fijación del EPS y acabado con pintura látex.

De esta forma, las transmitancias térmicas de los muros se reducirían a valores admisibles según IRAM $\left(0,65 \mathrm{~W} / \mathrm{m}^{2 \circ} \mathrm{K}\right.$ para el muro tipo $A$ y $0,69 \mathrm{~W} / \mathrm{m}^{2}{ }^{\circ} \mathrm{K}$ para el muro tipo $\left.\mathrm{A}\right)$ y el "G" resultaría en $1,94 \mathrm{~W} / \mathrm{m}^{3 \circ} \mathrm{K}$. La carga térmica anual "Q" tendría un valor de $249535 \mathrm{Kwh}$ (o su equivalente de $21328 \mathrm{~m}^{3}$ de $\mathrm{GN}$ ), obteniéndose una disminución del consumo total del orden de $\mathbf{8 , 5} \%$. 
Análisis y propuesta de mejoramiento energético, económico y de habitabilidad en la edificación educativa pública de la UNS

Fernández, Rodríguez, Genovese- Colaboradores: Rasquete, Obiol

Alternativa 3: la implementación combinada de las dos alternativas antes descritas, junto con la aislación de la cubierta a través de la incorporación de lana de vidrio (densidad estándar) de $70 \mathrm{~mm}$ por encima del cielorraso, podría reducir el consumo energético en un valor aproximado a un tercio, a la vez de alcanzar los valores de transmitancia térmica admisibles según la norma IRAM y exigidos en la mencionada ley 13.059 de la provincia de Buenos Aires.

En consecuencia, estos valores para los muros serían de $0,65 \mathrm{~W} / \mathrm{m}^{2 \circ} \mathrm{K}$ (muro tipo A) y 0,69 $\mathrm{W} / \mathrm{m}^{2 \circ} \mathrm{K}$ (muro tipo B). El valor de las carpinterías sería de $3,20 \mathrm{~W} / \mathrm{m}^{2 \circ} \mathrm{K}$. Y el "K" de la cubierta sería de $0,43 \mathrm{~W} / \mathrm{m}^{2 \circ} \mathrm{K}$ para invierno y $0,40 \mathrm{~W} / \mathrm{m}^{2 \circ} \mathrm{K}$ para verano. El "G" resultaría en $1,43 \mathrm{~W} / \mathrm{m}^{3 \circ} \mathrm{K}$ (valor aún por debajo del admisible según la Norma: $1,18 \mathrm{~W} / \mathrm{m}^{3} \mathrm{~K}$ ). La carga térmica anual " $\mathrm{Q}$ " tendría un valor de 184582 Kwh (o su equivalente de $15776 \mathrm{~m}^{3}$ de $\mathrm{GN}$ ), obteniéndose una disminución del consumo total del orden de $32 \%$.

\section{Evaluación económica y síntesis}

A continuación, se detallan los costos iniciales de inversión para la ejecución de cada una de las alternativas propuestas, seguido del análisis de recuperación de la inversión.

\section{a) Costos iniciales de inversión}

Los valores de los materiales fueron estimados como promedio, sin IVA, de precios de 3 proveedores locales (Bahía Blanca). Mientras que los costos de mano de obra fueron estimados según valores UOCRA (convenio tablas salariales, julio 2021, oficiales y ayudantes).

-Alternativa 1 (Sumatoria total de superficie vidriada $=243,5 \mathrm{~m}^{2}$ )

Total Materiales $\rightarrow 19.981 \$ / \mathrm{m}^{2}$

Total Mano de Obra $\rightarrow 1.598 \$ / \mathrm{m}^{2}$

Costo Final (Mat. + M. de O.) $\rightarrow 19.981 \$ / \mathrm{m}^{2}+1.598 \$ / \mathrm{m}^{2}=\mathbf{2 1 . 5 7 9} \$ / \mathrm{m}^{2}$

Costo Alternativa $1 \rightarrow 21.579 \$ / \mathrm{m}^{2} \times 243,5 \mathrm{~m}^{2}=\$ \mathbf{5 . 2 5 4 . 4 8 6 , 5}+$ IVA

-Alternativa 2 (Superficie total a intervenir, según cómputo métrico $=478,5 \mathrm{~m}^{2}$ )

Total Materiales $\rightarrow 1390,74 \$ / \mathrm{m}^{2}$

Total Mano de Obra $\rightarrow 1.486,99 \$ / \mathrm{m}^{2}$

Costo Final $\left(\$ / \mathrm{m}^{2}\right)$ (Mat. + M. de O.) $\rightarrow 1390,74 \$ / \mathrm{m}^{2}+1.486,99 \$ / \mathrm{m}^{2}=\mathbf{2 . 8 7 7 , 7 3} \$ / \mathbf{m}^{2}$

Costo Alternativa $2 \rightarrow 2.877,73 \$ / \mathrm{m}^{2} \times 478,50 \mathrm{~m}^{2}=\$ 1.376 .993,81+$ IVA

-Alternativa 3 (Superficie total a intervenir en la cubierta, según cómputo métrico $=466,34 \mathrm{~m}^{2}$ )

Total Materiales $\rightarrow 844,82 \$ / \mathrm{m}^{2}$

Total Mano de Obra $\rightarrow 1.007,60 \$ / \mathrm{m}^{2}$

Costo Final $\left(\$ / \mathrm{m}^{2}\right)$ (Mat. + M. de O.) $\rightarrow 844,82 \$ / \mathrm{m}^{2}+1.007,60 \$ / \mathrm{m}^{2}=\mathbf{1 . 8 5 2 , 4 2} \$ / \mathbf{m}^{2}$

Costo estimado aislación cubierta $\rightarrow 1.852,42 \$ / \mathrm{m}^{2} \times 466,34 \mathrm{~m}^{2}=\$ \mathbf{8 6 3 . 8 5 7 , 5 4}$ + IVA

Costo Alternativa $3 \rightarrow \$ 5.254 .486,5+\$ 1.376 .993,81+\$ 863.857,54=7.495 .337,85+$ IVA

En síntesis, la propuesta de mejoramiento termo-energético de la alternativa 1 redujo un $45 \%$ las pérdidas en aberturas, con un costo inicial de inversión (CII) de \$5.254.486,50+IVA. La alternativa 2 redujo un $38 \%$ las pérdidas por muros, con un CII de $\$ 1.376 .993,81+$ IVA. Mientras que la alternativa 3 combina las dos anteriores, más un $75 \%$ de reducción en las pérdidas por cubierta, sumando un Cll total de $\$ 7.495 .337,85+$ IVA.

b) Análisis de recuperación de la inversión 
Análisis y propuesta de mejoramiento energético, económico y de habitabilidad en la edificación educativa pública de la UNS

Fernández, Rodríguez, Genovese- Colaboradores: Rasquete, Obiol

En la figura 3 se grafican las estimaciones de los costos totales, en función del tiempo. Como se expresó previamente en la metodología, para la situación original (base) se calcula la acumulación anual del Costo Operativo (CO), sometido a una tasa de inflación anual y se resta el valor de renta (como una ganancia potencial) producto de invertir el capital no utilizado para el reciclado, por ejemplo, en un plazo fijo. Los costos totales de las alternativas de mejoramiento 2 y 3 , se calculan como la sumatoria del CO sometido a una tasa de inflación anual, con el Costo Inicial de Inversión (CII), sometido a un valor de renta (entendido este como un costo por perder el potencial de ganancia).

Para el cálculo de los costos anuales, se considera el costo del gas natural (GN) en $13,40 \$ / \mathrm{m}^{3}$ (valor para consumos mayores a $1350 \mathrm{~m}^{3}$ mensuales, más un $20 \%$ estimado de cargos fijos. No se considera el IVA ni en el combustible ni en los costos de los materiales y mano de obra de las alternativas de mejoramiento.). A su vez estos valores son afectados por una tasa constante de inflación anual del 50\% (valor estimado como la media entre el valor oficial de 39,10\% y valores de fuentes privadas del orden del 60\%). Y un valor de renta del 22\% (media histórica de la tasa anual de plazo fijo del Banco de la Nación Argentina, entre 2015 y 2020).

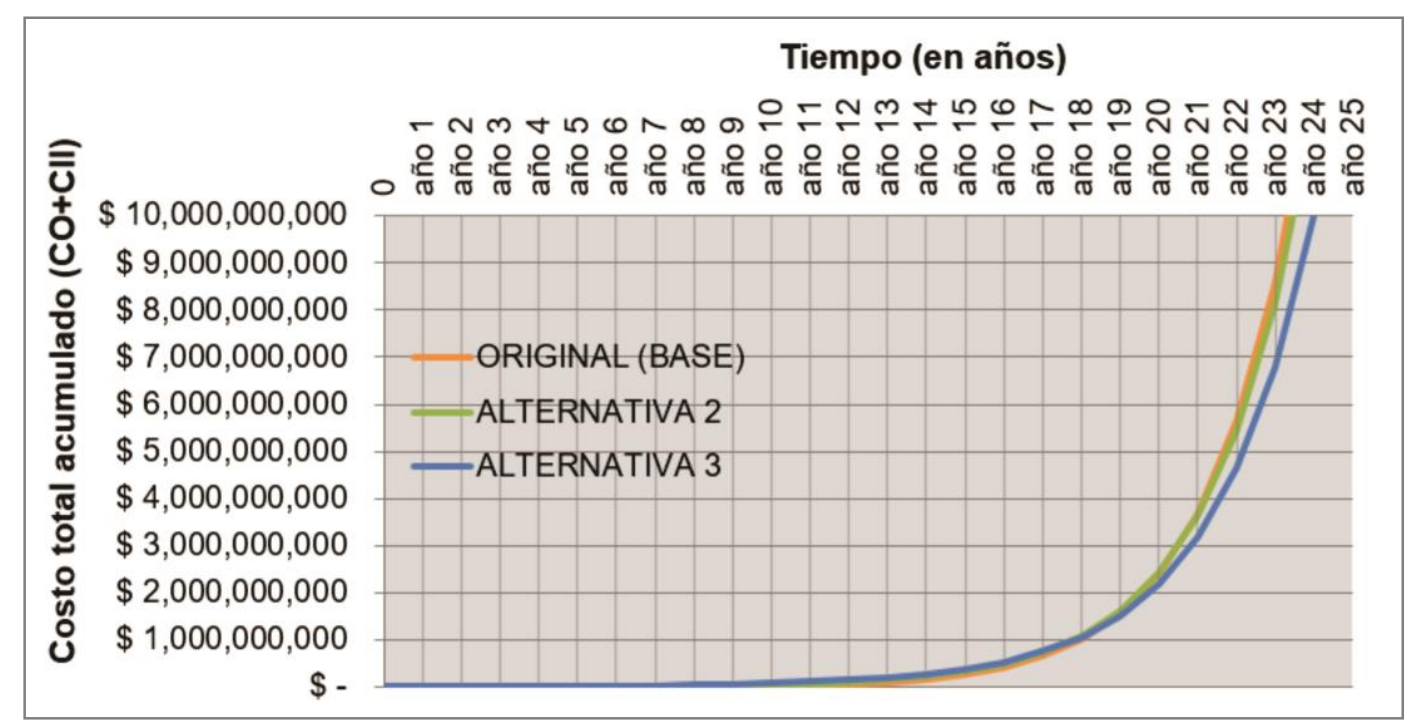

Figura 3. Gráfico de amortización del estado original y alternativas de mejoramiento 2 y 3 . Fuente: elaboración propia

\section{c) Síntesis}

En la tabla 2 se exponen los principales resultados del trabajo. Vale rescatar que los valores adoptados para la estimación del tiempo de amortización son parámetros que pueden variar considerablemente, según los supuestos de partida con que se construyan las proyecciones. Pero más allá del valor de costos de la energía, costos de inversión, inflación, renta, y del planteo de máxima o de mínima: en los escenarios más desfavorables, los tiempos de amortización oscilan en el orden de los 30 años; mientras que las condiciones más propicias para la aplicación de planes de eficiencia energética se estiman en el orden de 10 años o menos (RODRIGUEZ et al., 2017). Estos tiempos son perfectamente aceptables para acciones del Estado, más aún si consideramos que las mejoras en la habitabilidad, las reducciones de demanda energética y las emisiones de GEl son inmediatas. 
Análisis y propuesta de mejoramiento energético, económico y de habitabilidad en la edificación educativa pública de la UNS

Fernández, Rodríguez, Genovese- Colaboradores: Rasquete, Obiol

Tabla 2: Valores y resultados del estudio para situación original y propuestas de mejoramiento

\begin{tabular}{|c|c|c|c|c|c|c|}
\hline & $\begin{array}{l}\text { Consumos } \\
\text { (GN) }\end{array}$ & $\begin{array}{c}C O \\
\text { (año 2021) }\end{array}$ & $\begin{array}{c}C I I \\
\text { (año 2021) }\end{array}$ & $\begin{array}{l}\text { Ahorro } \\
\text { global }\end{array}$ & $\begin{array}{c}\text { Recuperación } \\
\text { de la } \\
\text { inversión }\end{array}$ & $\begin{array}{c}\text { Emisiones } \\
\text { de } \mathrm{CO}_{2}\end{array}$ \\
\hline $\begin{array}{l}\text { Original } \\
\text { (Base) }\end{array}$ & $23.326 \mathrm{~m}^{3}$ & $\$ 400.206,98$ & - & - & - & $46,22 \mathrm{~kg}$ \\
\hline Alternativa 1 & $20.757 \mathrm{~m}^{3}$ & $\$ 356.130,34$ & $\$ 5.254 .486,5+\mathrm{IVA}$ & $11 \%$ & 23/24 años & $41,13 \mathrm{~kg}$ \\
\hline Alternativa 2 & $21.328 \mathrm{~m}^{3}$ & $\$ 365.927,06$ & $\$ 1.376 .993,81+$ IVA & $8,5 \%$ & 18 años & $42,26 \mathrm{~kg}$ \\
\hline Alternativa 3 & $15.776 \mathrm{~m}^{3}$ & $\$ 270.670,73$ & $\$ 7.495 .337,85+$ IVA & $32 \%$ & 19/20 años & $31,26 \mathrm{~kg}$ \\
\hline
\end{tabular}

\section{CONCLUSIONES}

En adición a lo expresado en el punto anterior, se reconoce que la aplicación de las propuestas de mejoramiento tecnológico-constructivo en la edilicia existente presentan beneficios para todos los actores involucrados: con mejoras inmediatas en las condiciones de confort higro-térmico; reducciones significativas en el costo operativo y la demanda energética, con sus consecuente disminución en las emisiones de gases de efecto invernadero; la posibilidad de reducir la potencia instalada de los equipos de climatización; y la potencialidad de aplicar tecnologías de conservación energética de fácil ejecución, con costos de inversión inicial relativamente bajos y amortizables en períodos mediatos, brindando proyecciones altamente favorables para la esfera de la gestión educativa pública, asegurando la calidad de habitabilidad, la salud edilicia y la reducción del costo de energía auxiliar y sus subsidios.

Importancia de la investigación

Ante el marco actual de emergencia energética, se destacan acciones significativas desde el sector público en relación al impacto y mejora del sector construido, tales como: Decreto Nac. 140/07 de Uso Racional y Eficiente de la Energía; Ley de Eficiencia Energética № 13059/03 de la Prov. de Buenos Aires (Decreto reglamentario 1030/10 y actualizaciones de la Norma IRAM); Ordenanza 8757 del municipio de Rosario, 2011; Ley 4458/12 de CABA para el Acondicionamiento Térmico en la Construcción de Edificios; Decreto Nac. 134/15 que declara la Emergencia Energética Nacional; Decreto 9/17 que declara el 2017 como "Año de las Energías renovables"; Proyecto de Ley Nacional S-2448/15 "Régimen de fomento nacional para la generación de energía distribuida a partir de fuentes renovables"; Norma IRAM 11900 (2017) sobre prestaciones energéticas en vivienda (etiquetado energético respecto de energía en climatización, agua caliente sanitaria, energía solar térmica y fotovoltaica e iluminación).

Estos marcos normativos y legales brindan un apoyo sólido para afianzar bases en la conservación del recurso energético a partir de mejorar la tecnología constructiva de los edificios públicos; obteniéndose mejoras en dos direcciones principales: por un lado, incrementando las condiciones de habitabilidad y confort higrotérmico. Por otro, aumentando la eficiencia en el uso del recurso económico, por la disminución de la demanda energética a partir del mejoramiento tecnológico-constructivo (que habilita una recuperación de la inversión inicial en períodos mediatos) (RODRIGUEZ et al., 2017).

\section{REFERENCIAS BIBLIOGRÁFICAS}

Cartier, E. N. (2017). Apuntes para una teoría del costo. Colección Académica La Ley, Bs. As.

Discoli, C. (2009). Metodología para el diagnóstico urbano-energético-ambiental en aglomeraciones intermedias. El Caso del Gran La Plata. Libro elaborado sobre la Tesis doctoral del autor. Editorial Universitaria de La Plata, La Plata.

Rodríguez, L.; Díscoli, C.; Martini, I. (2017). Criterios y métodos para el reciclado edilicio con eficiencia energética. La Plata: Servicop. 